\title{
Sentinel lymph node in cervical cancer: time to move forward
}

\author{
Vincent Balaya $^{1} \wedge$, Benedetta Guani ${ }^{1 \wedge}$, Basile Pache ${ }^{1 \wedge}$, Yves-Gérard Durand ${ }^{1}$, Hélène Bonsang-Kitzis ${ }^{2}$, \\ ${\text { Charlotte } \operatorname{Ngô}^{2} \wedge}^{2}$, Patrice Mathevet ${ }^{1 \wedge}$, Fabrice Lécuru $^{3 \wedge}$
}

${ }^{1}$ Département Femme-Mère-Enfant, Centre Hospitalier Universitaire Vaudois, Lausanne, Switzerland; ${ }^{2}$ Department of Gynecologic and Oncologic Surgery, Hôpital Privé des Peupliers, Paris, France; ${ }^{3}$ Breast, Gynecology and Reconstructive Surgery Unit, Institut Curie, Paris, France

Contributions: (I) Conception and design: V Balaya, P Mathevet, F Lécuru; (II) Administrative support: V Balaya, B Guani, P Mathevet, F Lécuru; (III) Provision of study materials or patients: V Balaya, B Guani, P Mathevet, F Lécuru; (IV) Collection and assembly of data: V Balaya, B Guani, B Pache, YG Durand, H Bonsang-Kitzis, C Ngô; (V) Data analysis and interpretation: V Balaya, B Guani, B Pache, YG Durand, H Bonsang-Kitzis, C Ngô; (VI) Manuscript writing: All authors; (VII) Final approval of manuscript: All authors.

Correspondence to: Vincent Balaya. Département Femme-Mère-Enfant, Service de Gynécologie, Centre Hospitalier Universitaire Vaudois, Avenue Pierre-Decker 2, CH- 1011 Lausanne, Switzerland. Email: Vincent.Balaya@chuv.ch.

\begin{abstract}
In early-stage cervical cancer, lymph node status is of paramount importance to determine the best therapeutic strategy and is one of the most important prognostic factors of survival. According to main international guidelines, pelvic full lymphadenectomy is recommended for lymph node staging. Sentinel lymph node (SLN) biopsy is an accurate method for the assessment of lymph nodal involvement and has been suggested instead of systematic pelvic lymph node dissection (PLND). The SLN technique requires a learning-curve to be well performed. Combined detection with technetium-99 and blue dye has been widely used but the recent introduction of indocyanine green (ICG) is of growing interest since it could improve SLN detection. SLN biopsy offers a more accurate anatomical staging by finding potential metastatic nodes outside of usual lymphadenectomy areas. SLN biopsy improves the diagnostic value of lymph node staging with ultrastaging and detection of low-volume nodal metastases [isolated tumor cells (ITCs) and micrometastases]. Appropriate selection of patient and minimal training combined with some simple rules may guarantee a low false negative rate. Several studies have shown that SLN mapping in these patients is feasible, with excellent detection rates and sensitivity. Less-radical lymph node dissection decreases the associated morbidity of PLND, especially the risk of lower-limb lymphoedema, which severely affects patient quality of life. Some points are still subject to debate such as the low accuracy of intraoperative SLN status assessment by frozen section and the impact of micrometastasis on prognosis. Although international guidelines consider SLN biopsy as an alternative to PLND, SLN biopsy alone is not the gold-standard yet due to lack of prospective evidence on long-term oncological safety. The 3 ongoing prospective trials SENTIX, PHENIX and SENTICOL III will most probably give an answer to these issues.
\end{abstract}

Keywords: Cervical cancer; sentinel lymph node (SLN); ultrastaging; SENTICOL; lymphatic mapping

Submitted Jan 12, 2021. Accepted for publication Jan 27, 2021.

doi: $10.21037 / \mathrm{cco}-21-5$

View this article at: http://dx.doi.org/10.21037/cco-21-5

^ ORCID: Vincent Balaya, 0000-0002-7561-239X; Benedetta Guani, 0000-0003-4498-3734; Basile Pache, 0000-0003-0969-7345; Hélène Bonsang-Kitzis, 0000-0002-5594-2965; Charlotte Ngô, 0000-0002-6225-8803; Patrice Mathevet, 0000-0003-2782-4504; Fabrice Lécuru, 0000-0002-4071-3932. 
With nearly 570,000 new cases/year and 310,000 deaths/year, cervical cancer is the second most frequent cancer in women and the third leading cause of cancer-related deaths in females (1). The incidence and the mortality are a matter of concern in developing countries where screening programs are not always available. Additional measures such as patient education, changes in sexual behaviour and the introduction of HPV-vaccination may decrease the impact of this cancer $(2,3)$. It is important to emphasize that more than $54 \%$ of patients diagnosed are younger than 50 years (4).

Based on the prospective data of SENTICOL I and LACC trial, good oncologic outcomes after surgery have been reported for early-stage cervical cancer with a diseasefree survival of $90.6 \%$ at 3 years and $96.5 \%$ at 4.5 years respectively and an overall survival of $96 \%$ and $99 \%$, respectively $(5,6)$. For this subset of patients, lymph node status is a major prognostic factor since five-year diseasefree survival falls from $88 \%$ to $57 \%$ in case of lymph node metastasis (7) and has been recently included in the 2018 current International Federation of Gynecology and Obstetrics (FIGO) classification (8). Moreover, lymph node assessment is of paramount importance to determine the most appropriate therapeutic strategy for patients with early-stage cervical cancer, since positive pelvic node may require para-aortic lymphadenectomy and chemoradiotherapy rather than radical surgery.

According to main international guidelines, full pelvic lymphadenectomy is recommended for lymph node staging $(9,10)$. Nevertheless, this surgical procedure is associated with significant morbidity, especially a higher risk of lower limbs lymphedema $(11,12)$. The current trend in cervical cancer management is focused on less aggressive strategy without jeopardizing oncologic outcomes. Introduced first in breast cancer (13) and vulvar cancer (14), the sentinel lymph node (SLN) technique has been applied to early-stage cervical cancer within this logic and several publications have demonstrated the concept, the feasibility and the reliability in this indication (15-20). Based on European and American guidelines, SLN biopsy without additional lymphadenectomy is considered as acceptable for 2018 FIGO IA1 with lymphovascular space invasion (LVSI) and IA2 stages $(9,10)$. On the other hand, SLN biopsy alone is not recommended without systematic pelvic lymphadenectomy for lymph node staging for IB1 stage except in the context of prospective clinical trials according to the ESGO/ESTRO/ESP guidelines (9) whereas SLN biopsy could be considered for these cases according to the 2019 NCCN guidelines (10).
In this article, we review the current evidence on SLN biopsy concept in cervical cancer to determine whether SLN biopsy might be the gold-standard for lymph node staging.

\section{Rational}

Lymph node staging consisting in preoperative imaging and intraoperative sampling is mandatory for choosing the best therapeutic strategy (9). Pelvic lymphadenectomy sought to provide prognostic information that would indicate adjuvant radiotherapy to reach a loco-regional control of the disease. However, some substantive changes have to be considered about positive nodes in the modern era compared to old previous report (21-23). Size of metastatic node is usually small and is smaller than $2 \mathrm{~mm}$ in $22 \%$ to $60 \%$ of positive node patients (24-26). Given that most of metastatic nodes measured less than $10 \mathrm{~mm}$ (27), pelvic MRI and PETCT lack of sensibility and fail to identify positive-node patients (28). Nonetheless, the recent results of UTERUS-11 study did not show a difference in diseasefree survival between surgical and clinical staging in patients with locally advanced cervical cancer (29).

In addition, the mean rate of positive nodes in patients with early-stage cervical cancer is lower than $20 \%(15,18,26)$ and the number of metastatic nodes per patient ranges from 1 to 3 , with only one node being involved in near half of pN1-patients $(30,31)$. The full pelvic lymphadenectomy, consisting in removing at least 15 nodes which are analysed by one cut level and standard pathology, raised two main pitfalls: the induced postoperative morbidity and its efficiency. As previously stated, near $80 \%$ of patients who are free of nodal metastases would undergo useless pelvic lymphadenectomy and therefore would ensure associated complications such as longer operative time, blood loss, postoperative infection, lymphocyst and lower limbs lymphedema $(12,32)$. Although pelvic lymphadenectomy is completed, $10 \%$ to $15 \%$ of patients initially considered as N0 will experience cancer recurrences in the lymphatic area, due to the presence of atypical lymphatic drainage pathway (33). A targeted lymph node sampling appears more appropriate and SLN biopsy addresses particularly to this issue.

The SLN is defined as the first draining node of a solid tumor and represent the status of regional and downstream nodes. This concept applies to tumor with a sequential lymphatic drainage and a low rate of nodal involvement. The selective removal of these SLNs aims to ensure the 

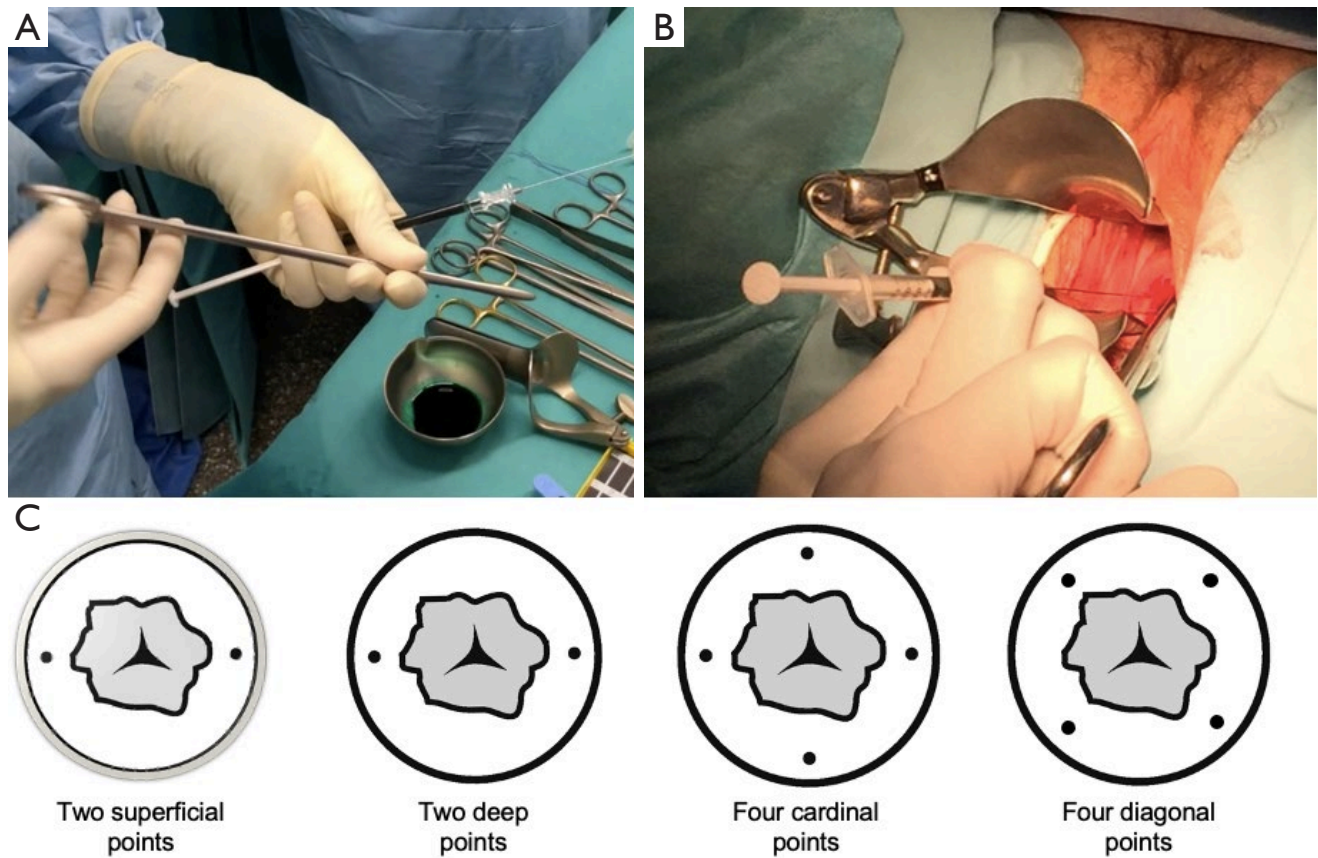

Figure 1 Colorimetric tracer injection technique. (A) Syringe preparation; (B) Injection through vaginal access; (C) Different sites of injection.

absence of lymphatic tumor spread in the first draining node and thus to predict the absence of distant lymphatic metastases in the secondary nodes. The SLN biopsy is a targeted sampling which should provide the same diagnostic information as the pelvic lymphadenectomy ( $\mathrm{pN} 0$ or $\mathrm{pN} 1$ ) with less morbidity and without compromising oncologic outcomes.

\section{SLN biopsy procedure}

\section{Injection technique}

According to the ESGO guidelines, SLN mapping might be performed by a combined technique of blue dye (patent blue, isosulfan blue or lymphazurin) in association with radiocolloid Technetium $99 \mathrm{~m}\left({ }^{99 \mathrm{~m}} \mathrm{Tc}\right)$ or by fluorescent dye alone [indocyanine green (ICG)] (9). Blue dye alone should be avoided and the association with isotopes may improve sensibility to detect SLN $(34,35)$.

The radioactive tracer colloidal rhenium sulfide labeled with technetium $\left({ }^{99 \mathrm{~m}} \mathrm{Tc}\right)$ is mainly used. Two protocol injection are feasible: a long or a short. In case of longprotocol, a dose of $120-\mathrm{MBq}$ is injected the day before surgery with a delay shorter than 15 hours. In case of shortprotocol, a dose of $60-\mathrm{MBq}$ is injected morning of surgery.
Whatever the type of protocol, cooperation with the nuclear medicine department is essential. The injection is performed in healthy tissue at the periphery of the tumor or of the conisation scar. By using a 25-gauge needle, submucosal injections are performed into two (at 3 and 9 o'clock) or four points at 5-10 mm-depth (Figure 1). To guide the surgeons during the surgical procedure, a preoperative lymphoscintigraphy may be done after the isotope injection ( 1 to 3 hours in case of short protocol or 3 to 5 hours in case of long protocol). Detection rate and diagnostic performance are not affected by either the two protocols $(36,37)$. Due to a better resolution, the single photon emission computed tomography (SPECT-CT) provides more accurate information than lymphoscintingraphy alone on SLN topography and improves surgical staging (38).

Two types of colorimetric tracer can be used: blue dye or ICG. Using ICG require specific near-infrared detection system (for both open surgery and minimally invasive surgery) to visualize the fluorescence, whereas blue dye does not require any specific material since it can be seen with the naked eye. Both colorimetric tracers can be used at the same time (39). Usually, $2 \mathrm{~mL}$ of $2.5 \%$ blue dye are diluted in $2 \mathrm{~mL}$ of saline or $25 \mathrm{mg}$ of ICG are diluted in $10 \mathrm{~mL}$ of glucose solution. Some authors suggested to inject 
concomitantly India ink to identify SLN in the parametrium at the final pathologic examination (40).

The injection of the colorimetric tracer is performed at the beginning of the surgical procedure following the same technique as described for radiocolloid injection. Practically, the injection can be done right after the induction of general anaesthesia, but due to the rapid migration of the tracer, this injection should be performed at the earliest after the surgical positioning of the patient in order to decrease the false negative results by identifying non-SLN instead the true SLN. In addition, late injection enhances to visualize intraoperatively dye migration until the true SLN. One millilitre of blue dye or ICG is injected into 2 or 4 points in the cervix (Figure 1). Higher injection volume should be avoided due to the risk of dye spillage in the surrounding tissue which may impair the surgical dissection. By contrast, a second injection can be performed during the surgery in case of unilateral detection (41).

A particular attention should be paid for a meticulous injection technique in order to decrease detection failure $(42,43)$. Some technical problems can occur with the cervical injection such as wrong injection site (too lateral or in the external cervical ostium), the presence of Nabothian cysts, tiny atrophic cervix in menopausal women, bleeding, distorted cervical anatomy, or lack of experience. In case of larger tumors and bulky cervix, tracer injection technique is more difficult due to the frequent presence of a dense and necrotic central core that may inhibit tracer diffusion (44). Appropriate modification of tracer injection technique may overcome this difficulty by using longer needle, bigger volume of blue dye and injection in the residual stroma around the tumor (45).

\section{Surgical dissection}

Surgery can be performed by minimally invasive approach (laparoscopy or robotic-assisted laparoscopy) or by open surgery. Most of published data are derived from series in which surgery was mainly performed by minimally invasive approach but the results of the LACC trial will certainly induce a paradigm shift in favour of open approach (6).

The intraoperative detection starts by exploring the abdominal and pelvic cavities over an intact peritoneum in order to look for stained and/or radioactive nodes detected by the Gamma probe. An adhesiolysis may be necessary in case of previous pelvic surgery. The absence of grossly suspicious nodes has to be verified even if this eventuality is less likely with an adequate preoperative imaging. The dye migration has to be followed in regard with the parametrium, internal iliac area, interiliac area, external iliac area, common iliac area and paraaortic area as described by Marnitz et al. (46) (Figure 2).

Second, the entire retroperitoneal space should be opened along the external iliac vessels with pararectal and paravesical fossa as well. Anatomical landmarks such as the umbilical artery, the ureter, the obturator nerve have to be identified (Figure 3). This approach permits to see the early drainage from the cervix through the parametrium by following the dye progression in the channels before any node is taken to ensure that the true draining SLN is identified and not missed (Figure 4). SLN are usually found in the external iliac and interiliac area (47). The SLN mapping may be guided by the information provided by the lymphoscintigraphy. Even if the false negative rate may be limited by increasing the number of SLNs sampled especially in teams with low experience, not all detected nodes (blue and/or hot SLN) should be removed but only the first draining node in the channel pathway has to be removed and labeled as SLN (Figure 5). This step is crucial to correctly identify the true SLN and not to confuse with non-SLN which correspond in fact to distal migration of tracer beyond the true SLN. Nonetheless, more SLNs can be sampled in case of truly separate channels which may correspond to distinct lymphatic drainage pathway (Figure 6). If no SLNs are found in the external iliac, interiliac and common iliac area, dissection of the promontory area and paraaortic area should be performed to avoid missing SLN in atypical topography (48). Whatever the type of technique used, a bilateral detection should be obtained (at least one SLN in each hemi-pelvis).

In case of unilateral detection, extensive dissection of the retroperitoneal space has to be performed carefully due to the risk of diffuse smearing induced by an excessive manipulation. A second injection of colorimetric tracer might be helpful. According to MSKCC algorithm, if no SLNs are found, an ipsilateral pelvic lymphadenectomy should be performed (17). Caution should be paid to swollen lymphatics channel which might be mistaken with SLN and are responsible of $40 \%$ of detection failure (43). Detected SLNs are removed selectively and sent for frozen section examination (FSE) after ex vivo palpation.

Finally, the absence of residual in vivo radioactivity and the absence of residual fluorescence are checked. The opening of the paravesical and pararectal space enhance to individualize the parametrium and to begin the radical hysterectomy. At this step, SLNs in parametrium can be 

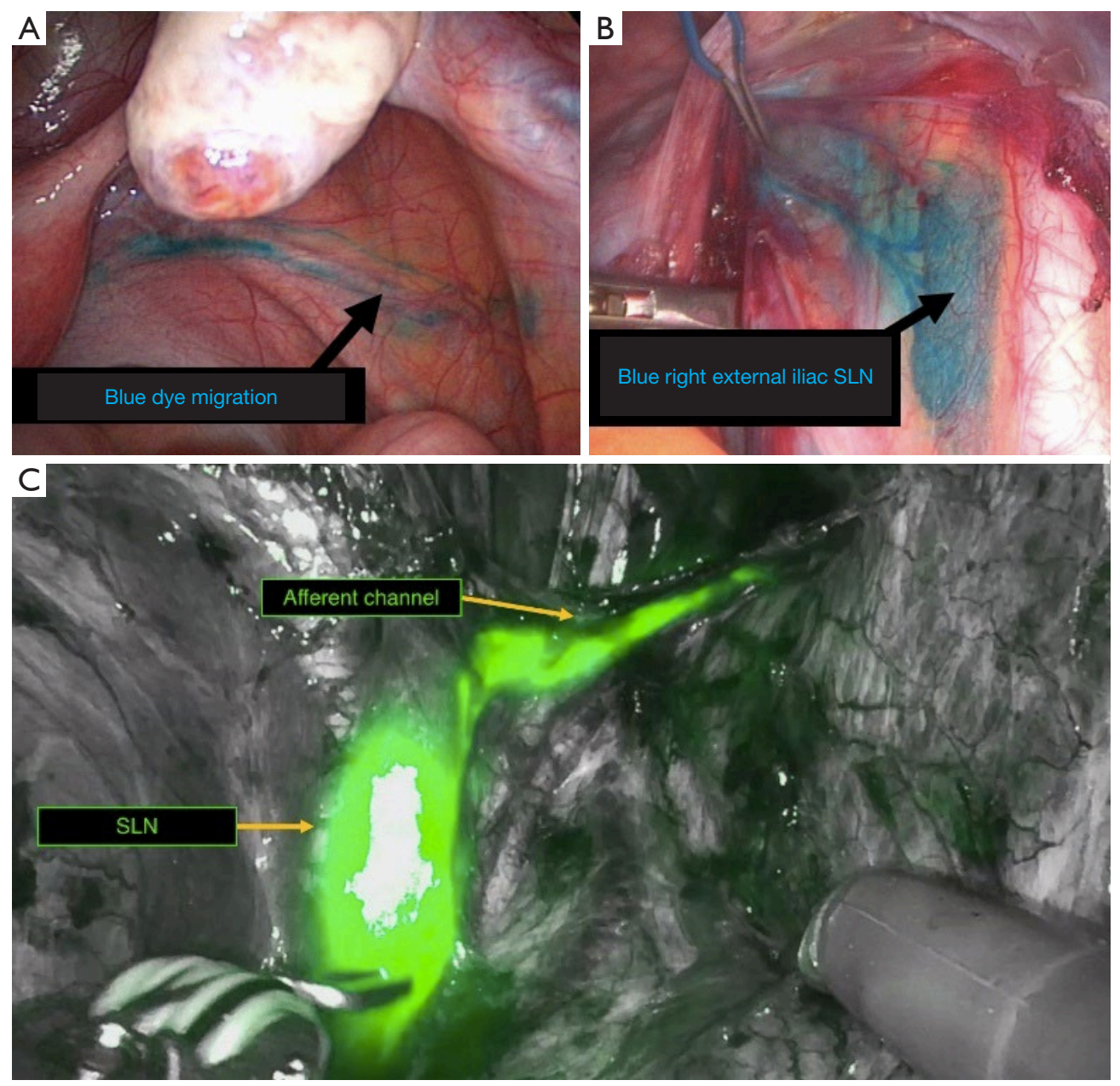

Figure 2 Intraoperative view of blue tracer migration (A,B) and ICG migration (C). SLN, Sentinel lymph node; ICG, indocyanine green.

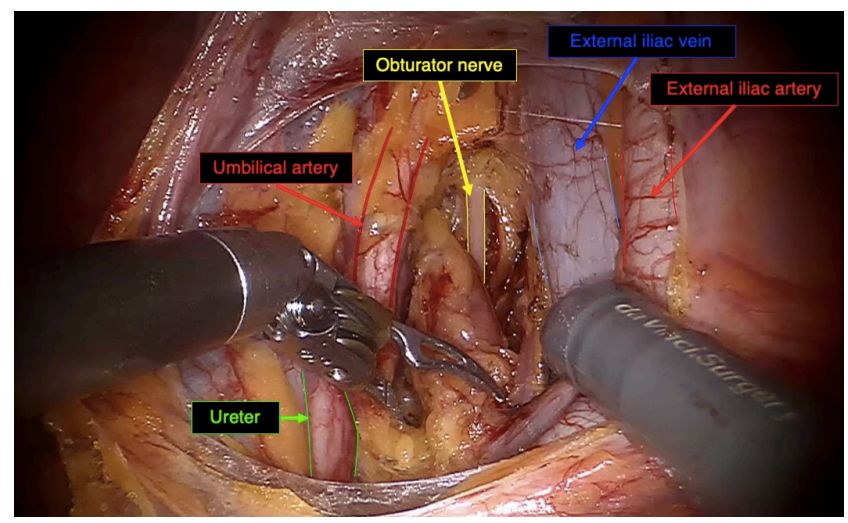

Figure 3 Laparoscopic intraoperative view of the right ilioobturator fossa with anatomical landmarks.

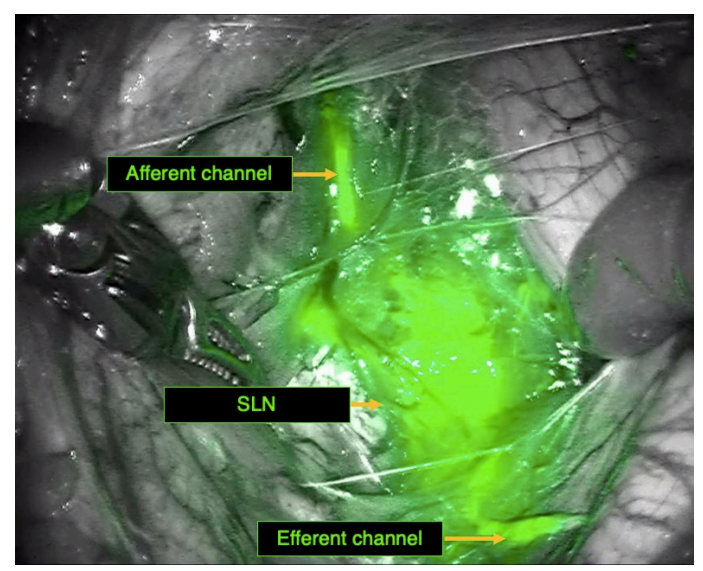

Figure 4 Laparoscopic intraoperative view of the right ilioobturator fossa with fluorescent afferent lymphatic channel, fluorescent SLN and fluorescent efferent lymphatic channel. SLN, Sentinel lymph node. 


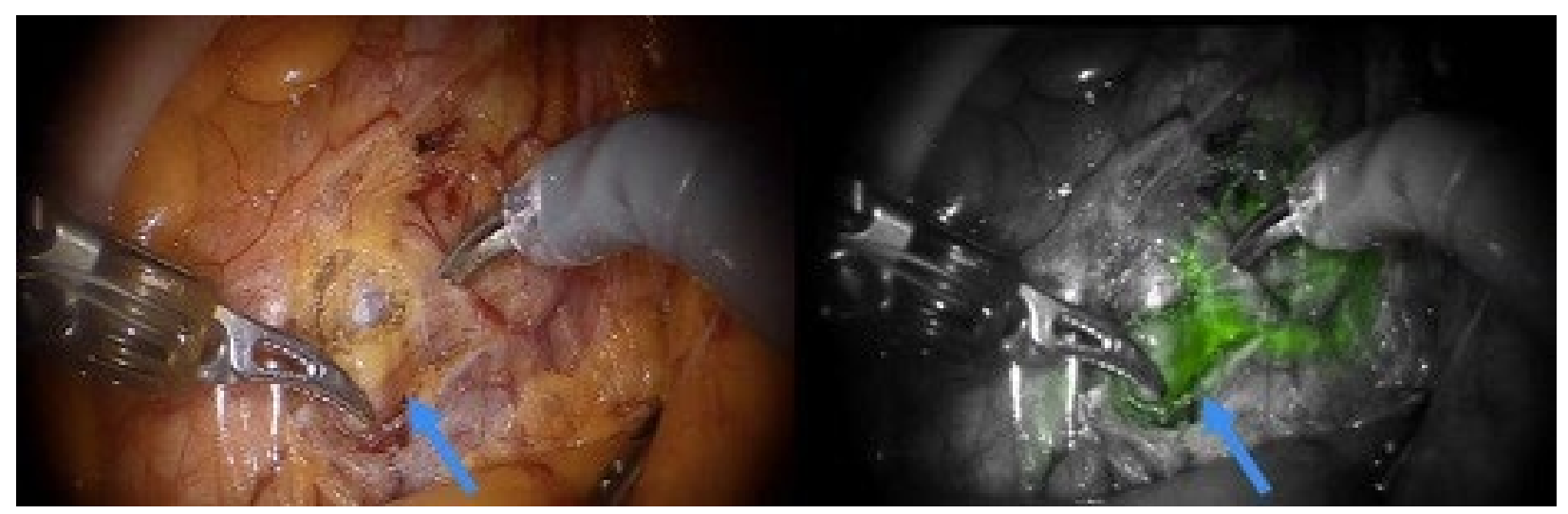

Figure 5 Sampling of fluorescent SLN (blue arrow). SLN, Sentinel lymph node.

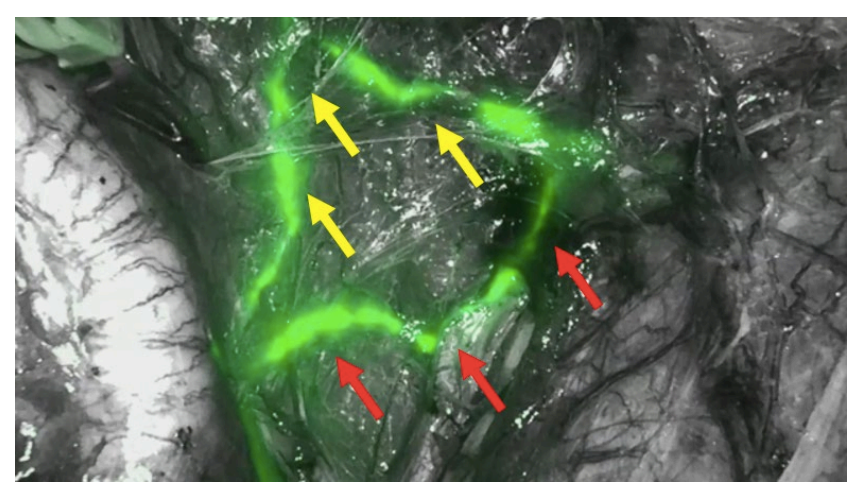

Figure 6 Intraoperative view of the left ilio-obturator fossa with two distinct lymphatic channels (yellow and red arrows).

found and have to be reached.

These different steps have to be respected to ensure the reproducibility and the reliability of the technique. This surgical methodology may be implemented in a check-list of "quality-assurance" (Table 1) (49).

\section{Experience and learning-curve}

Efficient cervical injection, intraoperative detection and accurate node sampling require a learning-curve which may be done by performing systematic SLN biopsy before each pelvic lymphadenectomy (for cervical or endometrial cancer) under the control of an experienced operator.

This concept of learning-curve has been widely described in the literature $(15,45,50-52)$. In their cohort of 55 patients, Plante et al. have shown a better SLN identification rate in the last 15 cases compared to their 28 first cases $(93 \%$ vs. $51 \%, \mathrm{P}<0.01)$ and concluded that experience in SLN
Table 1 Checklist "quality assurance" for SLN biopsy in cervical cancer

Preoperative

- Clinical stage $\leq \mathrm{IIA} 1$

- Abdominopelvic MRI: Cervical tumor size $<30 \mathrm{~mm}$ with intact parametria

- No suspicious pelvic or paraaortic nodes (small axis $\geq 10 \mathrm{~mm}$ and morphologic criteria)

- Training for the cervical injection of isotopic tracers

- Performance of lymphoscintigraphy (or SPECT) and appropriate training for the interpretation of these imaging modalities

Intervention

- Physical exam under general anesthesia: Clinical stage $\leq$ IIA1

- Training for the cervical injection of colorimetric or fluorescent dye

- Training in the exploration and dissection of SLN

- Bilateral SLN detection (if unilateral detection, contralateral hemipelvic lymphadenectomy)

Pathology

- Training on the performance of frozen section analysis of SLN

- Training on the performance of ultrastaging of SLN

SLN, sentinel lymph node.

detection increased significantly with time (15). This acquired experience had an impact in the improvement of SLN detection in subgroup of patients with higher risk of SLN detection failure such as patients with larger tumor size (45) and obese patients (52). According to Khoury- 
Collado et al., the cut-off for learning curve in SLN biopsy in endometrial cancer should be fixed at 30 cases (51). In an ancillary analysis of SENTICOL I and SENTICOL II cohorts, bilateral detection rate was significantly higher in SENTICOL II cohort (2009-2012) than in SENTICOL I cohort (2005-2007), respectively $83.5 \%$ vs. $75 \%$, $\mathrm{P}=0.04$ (42). Moreover, centre with low surgical skills ( $\leq 1$ case/year) had significantly lower bilateral detection rate than intermediate-skill centre (1-5 cases/year) and high-centre (>5 cases/year), $56.3 \%$ versus $78.3 \%$ and $83.1 \%$ respectively $(\mathrm{P}=0.02)$ (42).

As demonstrated in ovarian cancer, treatment in expert centre with a high-volume of surgical procedures is a quality indicator with better outcomes $(53,54)$. Surgery should be performed by gynecologic oncologist and trained surgeon in a specialized team dedicated to management of gynecologic cancers (9).

\section{Impact of ICG}

As previously mentioned, the ESGO guidelines recommend for SLN mapping might be performed by a combined technique of blue dye with radiocolloid ${ }^{99 \mathrm{~m}} \mathrm{Tc}$ or by ICG alone (9). SLN detection using ICG and near-infrared fluorescence has been introduced in 2012 by Rossi et al. in cervical and endometrial cancer (55).

In a meta-analysis including 538 patients with cervical or endometrial cancer and comparing ICG to blue dyes with or without ${ }^{99 \mathrm{~m}} \mathrm{Tc}$, Ruscito et al. have shown that ICG improved significantly overall detection rate $(\mathrm{OR}=0.27,95 \% \mathrm{CI}$ : 0.15-0.50. $\mathrm{P}<0.0001)$ and bilateral detection rate (OR $=0.27,95 \%$ CI: $0.19-0.40 . \mathrm{P}<0.00001)$ compared to blue dye alone. There were no differences in overall and bilateral detection rates between ICG and association of blue dyes and Technetium-99m (56). Near-infrared imaging with ICG enhances deeper visualization of SLN through adipose tissue with a penetration of $1 \mathrm{~cm}$ which may explain that ICG provides better SLN detection rate compared to blue dye in obese patients $(57,58)$. In a randomized controlled trial comparing ICG to methylene blue in 132 patients with endometrial cancer, ICG increased significantly the SLN detection rate per hemipelvis of $26.5 \%$ compared to methylene blue (59). Recently, the prospective randomized FILM trial has shown better SLN detection rates with ICG compared to blue dye in patients with endometrial and cervical cancer (97\% vs. 47\%, $\mathrm{P}<0.0001)(60)$.

ICG have several advantages. This tracer is easier to use and does not require lymphoscintigraphy and no human or technical resources linked to a nuclear medicine department. In terms of cost, an ICG vial is cheaper than using Technetium-99m (61). Preoperative radiocolloid tracer injection may be painful whereas ICG is injected after the induction of general anaesthesia. Risk of allergies and anaphylactic shocks are higher with blue dye (62) than with ICG (63).

Based on these results, this technique is of growing interest and will surely become soon the agent of choice for a worldwide implementation because of its safety profile, its ease of use, its affordable low cost and its diagnostic performance. Although ICG is a fluorescent dye approved by the FDA (Food and Drug Administration) and the European Medicines Agency, it seems important to precise that ICG is used off-label for intratissular injection to date.

\section{Anatomic SLN mapping}

One of the interests of SLN technique is to highlight the atypical lymphatic drainage pathway in secondary lymphatic area that are not systematically dissected during routine lymphadenectomies $(33,64)$. This technique is well appropriate to early-stage cervical cancer since the tracer injection is performed just around the tumor and reveal not only the cervical lymphatic drainage but the tumor lymphatic drainage as well.

Although most of SLNs are located in the interiliac and external ILAC area, SLNs have also been described in "unexpected" territories such as sacral, promontory and paraaortic areas $(33,46-48)$. To describe SLN topography, Marnitz et al. suggested the following area classification by (46): paraaortic (area 1), common iliac (from the bifurcation of the aorta to the bifurcation of the iliac vessels, area 2), external iliac (along the external iliac vessels, area 3 ), interiliac (nodes medial to the external iliac artery and vein, in the obturator fossa and over the bifurcation of the common iliac artery, area 4), internal iliac (medial to the internal iliac vessels down to the uterine artery bifurcation, including the presacral nodes, area 5), and parametrium (nodes medial to the bifurcation of the uterine artery, area 6). Areas 3 and 4 are considered to be located in typical sites whereas the other areas (areas 1,2,5, and 6) are defined as atypical sites. In a meta-analysis of 27 articles which included 1301 patients with 3012 SLNs, Ouldamer et al. reported that $83.7 \%$ of SLNs were located in classic areas of the pelvis (obturator, external iliac, and internal iliac), $6.6 \%$ in the common iliac area, $4.3 \%$ in the parametrial area, $2.0 \%$ in the paraaortic area, $1.3 \%$ in the presacral area, 
$0.2 \%$ in the hypogastric area, $0.07 \%$ in the inguinal area, and $0.07 \%$ in the cardinal ligament area. Takeshita et al. described also SLNs in the circumflex iliac area (65). Even if most of metastases are located in the pelvic area, it was also described that positive lymph nodes were also found in gluteal, presacral, and paraaortic areas. Although the prevalence of involved nodes in these anatomical regions is low, this raises the question of the efficiency of systematic extended lymphadenectomy $(30,66)$. Moreover, the knowledge of the pelvic lymphatic anatomy is fundamental to understand the extension of lymphatic involvement.

Several studies have described the uterine and cervical lymphatic drainage anatomy (67-70). More recently, some authors used ICG in SLN mapping for endometrial cancer and provided a surgical anatomic classification $(41,71)$. They described: an upper paracervical pathway (UPP) following the uterine artery to draining medial external and/or obturator lymph nodes with a continued course lateral to the common iliac artery to the lateral precaval and paraaortic areas, a lower paracervical pathway (LPP) with through the sacrouterine ligament to the internal iliac and/ or presacral draining nodes before continuing medial to the common iliac artery to the medial paraaortic and precaval areas and an Infundibulo-pelvic pathway (IPP) with a course along the fallopian tube and upper broad ligament via the Infundibulo-pelvic ligament to its origin. These pathways are linked to finer lymphatics at the level of the cardinal ligaments, thereafter, dividing into distinct noncommunicating courses lateral and medial to the common iliac arteries with further drainage to the paraaortic area. The UPP and the LPP were the most frequent pathway. In patients with tumor size larger than $20 \mathrm{~mm}$, usual lymphatic drainage may be altered (33). Lymphatic vessels may be obstructed by cancer cells and it may result in a modification of tumor lymphatic drainage. Furthermore, large tumors can have a central necrotic part that may change the diffusion of colorimetric or/and radiocolloid tracers (44). Tumor cells migration could use other lymphatic drainage pathway than that run to interiliac or external iliac area. More SLNs are found in atypical area in nulliparous women (33). During the pregnancy, endometrial stromal cell decidualization may induce some modifications of the repartition of lymphatic vessels which result in changing lymphatic network $(72,73)$.

Atypical pathway are present in $25 \%$ of patients and are exclusive in $10 \%$ of patients (33). These atypical pathways might contain some isolated positive nodal metastases that would not be removed during routine pelvic lymphadenectomy. This technique may reduce the false- negative case of lymph node staging and residual disease missing which leads to an undertreatment of patients. The frequency and the positive nodes rate of each lymphatic area are similar to those described in systematic pelvic and paraaortic lymphadenectomies (23), demonstrating that the same anatomic information could be obtained with a less invasive technique. Moreover, the SLN technique facilitates the sampling of informative and representative nodes especially in atypical locations not covered by conventional lymphadenectomy.

\section{Diagnostic value}

The diagnostic value of SLN biopsy is one of the most critical aspects of this technique. It has been demonstrated that bilateral negative SLN strongly predicted the absence of pelvic node involvement (74,75). The main current challenge of this technique is to achieve maximal sensitivity and lowest false-negative rates (20). The false negativity rate is of paramount importance since the majority of nodenegative patients do not receive adjuvant treatment and the nodal recurrence worsens severely the prognosis. Based on simple criteria such as early stage (IA2, IB1, IIA1 2018 FIGO stage), no suspicious pre-, and per-operative lymph nodes, and bilateral negative SLNs after ultrastaging, the false-negative rate may be very low at $0.08 \%$ in eligible patients (18).

Due to its central topography, historic anatomic work has proven that the uterine cervix has in fact a bilateral lymphatic drainage (76) and both hemipelvises have to be considered regarding two distinct lymphatic drainage pathway. Bilateral SLN detection is therefore of utmost importance to be fully reliable $(74,75,77)$. However, bilateral SLN detection is obtained in $60 \%$ of cases only (18). In a large prospective cohort of 405 patients, lower bilateral SLN detection rate was associated with Age $\geq 70$ years, tumor size larger than $20 \mathrm{~mm}$ and Body-mass index higher than $30 \mathrm{~kg} / \mathrm{m}^{2}(42)$. As previously mentioned, a learningcurve for SLN biopsy is required and the experience of the surgeon is essential.

Applying the MSKCC algorithm that is based on bilateral detection and sampling of all labeled nodes appears as a pertinent strategy since the majority of involved nodes are more likely diagnosed from the SLN removed rather than the non SLN ones (17). Following an anatomicallybased surgical algorithm, a precise and careful dissection of the retroperitoneal space may help to find SLN in atypical localization (48). Moreover, the SLN status may be considered 
to predict the parametrial involvement and to determine the type of radical hysterectomy to be performed $(78,79)$.

\section{Histopathology}

\section{Frozen section}

The FSE aims to determine intraoperatively the SLN status. Current guidelines suggest to submit SLNs to FSE to decide if a hysterectomy is to be pursued during the same surgical intervention (9). When positive SLN is found at FSE, two decisions have to be made: on one hand, abandoning or performing the radical hysterectomy and on the other hand, abandoning or performing pelvic +/paraaortic lymphadenectomy (80). If a radical hysterectomy is performed in this setting, it has to be considered nonetheless that combined treatment should be avoided due to the increased morbidity of surgery associated with adjuvant radiotherapy $(9,81)$. The SLN status is particularly crucial in case of fertility-sparing procedure since trachelectomy should not be performed if SLN are involved. This selective nodal sampling is particularly appropriate for the patients with the lowest risk of node involvement who are potentially eligible to simple trachelectomy or conisation (82).

The accuracy of FSE constitutes one of the main limitations of the SLN biopsy because of its limited diagnostic value and low ability to detect low-volume metastases such as Isolated Tumor Cells (ITCs) and micrometastases (83-88). If all type of metastases are considered, sensitivity ranged from $42.3 \%$ to $87.5 \%$ and negative predictive value ranged from $89.7 \%$ to $98 \%$ whereas sensitivity ranged from $56.4 \%$ to $88.9 \%$ and negative predictive value ranged from $91 \%$ to $98.8 \%$ if ITCs were excluded $(84,85,87-90)$. These wide ranges of value in the literature might be explained by the poor sensitivity of FSE to detect low-volume metastases (ITCs and micrometastases), a better sensitivity for macrometastases and different SLN involvement rate in each population study $(84,89)$. Applying FSE in a population with less macrometastases and more low-volume metastases may lead to a decreased sensitivity of FSE. By simply bisecting the SLN along its long axis and staining with hematoxylin-eosin (HE), a non-negligible proportion of macrometastases would also be missed by FSE in $14.9 \%$ to $16.7 \%$ of cases $(84,90)$. Slicing 2 -mm intervals the SLN along the short axis may increase the accuracy of intraoperative SLN examination $(86,87)$.

Several other factors may affect the false-negativity rate of FSE. A meticulous and specific protocol of pathologic processing, a higher volume of cases and the pathology team expertise might improve this technique $(85,90)$. The FSE is more efficient in smaller tumor size $\left(<20 \mathrm{~mm}\right.$ or $\left.<20 \mathrm{~cm}^{3}\right)$ since the risk of low-volume metastases is higher in larger tumor $(84,90)$. The presence of LVSI and preoperative brachytherapy were also associated with false negativity of FSE $(84,90)$.

Clinical relevance by focusing on macroscopic suspicious nodes has to be determined (84). For grossly suspicious SLN, a single section seems appropriate and surrounding adipose tissue lied to the node should be spared to assess extranodal extension. The presence of enlarged nodes nonetheless had no impact on the accuracy of intraoperative examination SLN (84). By contrast, performing frozen section of grossly normal nodes is subject to debate and caution should be paid for small nodes. Levelling the blocks too deep may expose to the risk of loss of tissue for final pathologic examination and may adversely affects the quality of ultrastaging and immunohistochemistry (20). The accuracy of FSE might be improved by using alternative technique such as the one-step nucleic acid amplification (OSNA) technique (91) or HPV DNA testing (92). Recently, Bizzarri et al. have shown that OSNA technique was efficient to detect intraoperatively low-volume metastases in SLN (93).

\section{Ultrastaging}

The small number of sampled nodes during the SLN biopsy allows to use more sensitive processing techniques such as immunohistochemistry and polymerase chain reaction (PCR). Ultrastaging, combining serial sectioning and immunohistochemistry, enhances to detect low-volume such as ITCs $(<0.2 \mathrm{~mm})$ and micrometastases $(0.2-2 \mathrm{~mm})$ which are rarely found with one-cut section and conventional coloration. This strategy increases the prevalence of node-positive patients up to $15 \%$ (27) and improves the nodal staging, especially when SLN are detected in both hemipelvis (77). Moreover, performing ultrastaging and immunohistochemistry on non-SLN does not change the false-negative rate (94). Compared to OSNA technique, Santoro et al. highlighted that ultrastaging protocol had higher sensitivity and more reliability in prediction pelvic non-SLN status (95). SLNs bring also other valuable prognostic information. In patients with negative SLN, HPV-mRNA is a molecular marker for disseminated tumor cells and its detection in negative SLN is an independent 
prognostic factor of recurrence (92). In early-stage cervical, the SLN is the site of a pre-metastatic lymphangiogenesis (pre-metastatic niche) which lead to specific humoral immune response (96). In a near future, these immunologic factors will provide a more precise prognostic assessment compared to current surgicopathologic factors.

The clinical significance of low volume metastases (micrometastases and ITCs) remains unclear. According to the revised FIGO classification, the presence of micrometastases is classified as IIIC stage whereas ITCs might be noted without modifying the stage $(8,97)$. Few data are available on ITCs prognostic value but ITCs seem to have no impact on oncologic outcomes and do not change oncologic management (98). In a retrospective cohort of 894 patients, Horn et al. reported that patients with micrometastasis had a significant decreased 5-year disease-free survival compared to $\mathrm{N} 0$ patients (68.9 vs. $91.4 \%, \mathrm{P}<0.001)$ and micrometastasis were found to be an independent prognostic factor by multivariate analysis (25). In a multicentric retrospective cohort of 645 patients, Cibula et al. found that micrometastases in SLN was associated with a similar overall survival as macrometastases, whereas ITCs had no impact on overall survival (99). These results were concordant to those published by Colturato $e t$ $a l$. who have shown that micrometastasis was a significant risk factor for tumor recurrence $(\mathrm{P} \leq 0.030)(100)$.

However, these results are counterbalanced by most recent prospective studies $(5,26,101)$. In SENTICOL I cohort, no significant difference in disease-free survival between patients with positive or negative lymph nodes was found (91.6\% vs. $90.4 \%$, respectively; $\mathrm{P}=0.49)$. In addition, among the 8 patients with SLNs positive for micrometastases, only one had a vaginal recurrence. These preliminary results have to be interpretated with caution because ultrastaging was performed on SLN a few months after primary surgery and did not impact on adjuvant treatment decision. Nonetheless, these results were confirmed in the pooled analysis of SENTICOL I and II cohorts and patients with low-volume metastasis had similar disease-free survival (DFS) (92.7\%) to N0 patients (93.6\%) (26). In a subset of 19 patients with low-volume metastases (10 with micrometastases and 9 with ITCs), the authors concluded that adjuvant treatment did not seem to affect the recurrence rate $(\mathrm{P}=0.5)$ and disease-free survival was comparable to those reported in historical cohorts (101).

Due to the low prevalence of ITCs and micrometastases, prospective multicenter clinical trials seem hardly appropriate to confirm these data and a large collaborative network cooperation would be required to determine the risk associated with these low-volume metastases in cervical cancer and their subsequent management.

\section{Morbidity and quality of life}

Full pelvic lymphadenectomy results in a high postoperative morbidity, especially lower limbs lymphedema. This morbidity is increased when circonflex iliac nodes are removed beyond external iliac nodes (102), in patients with body-mass index higher than $25 \mathrm{~kg} / \mathrm{m}^{2}$ or in case of adjuvant radiotherapy $(103,104)$. One of the main clinical benefit of SLN biopsy is the reduced morbidity associated with pelvic lymphadenectomy. In a prospective study, Niikura et al. have shown a decrease of lower limbs lymphedema rate in patients with SLN biopsy alone compared to those who underwent full lymphadenectomy $(8,7 \%$ vs. $42 \%, \mathrm{P}=0.03)$ (12). In the SENTICOL II trial, SLN biopsy induced less lymphovascular morbidity compared to SLN biopsy associated with pelvic lymphadenectomy ( $31.4 \%$ vs. $51.5 \%, \mathrm{P}=0.0046)$ and less early postoperative neurologic symptoms (7.8\% vs. $20.6 \%$ $\mathrm{P}=0.01)$ with similar 3 -year disease-free survival (11). In a retrospective study comparing 1,188 patients with bilateral pelvic lymphadenectomy to 100 patients with bilateral SLN biopsy, Lennox et al. highlighted that SLN biopsy was associated with shorter operative-time (2 vs. $2.8 \mathrm{~h}, \mathrm{P}<0.001)$, less intraoperative blood loss (100 vs. $500 \mathrm{~mL}, \mathrm{P}<0.001)$, less transfusion ( $0 \%$ vs. $23 \%, \mathrm{P}<0.001)$, less post-operative infection ( $0 \% v s .11 \%, \mathrm{P}=0.001)$ and shorter hospital stay (32).

Considering the young age of patients and the good prognosis of early-stage, quality of life of patients is of paramount importance. According to SF-36 scale, Gianoni et al. demonstrated trend for a better quality of life and less severe leg heaviness and leg fatigue when a full pelvic lymphadenectomy was avoided (105).

\section{Perspective}

Despite all these data, SLN biopsy alone is not considered as gold-standard for SLN staging yet. The critical endpoint is the long-term survival of patients with bilateral negative SLN who are deemed to be free of nodal involvement and who did not receive adjuvant treatment. The survival and the oncologic outcomes of patients who underwent SLN biopsy alone are poorly reported in the literature. In the retrospective analysis of Lennox and Covens, there was 
no difference between patients who underwent bilateral SLN biopsy alone and those who underwent bilateral pelvic lymphadenectomy in terms of 2-year DFS (97\% vs. $95 \%$ ) and 5 -year DFS (93\% vs. 92\%) (32). In a posthoc analysis of both SENTICOL I and SENTICOL II cohorts presented at the ASCO 2020, the DFS patients with bilateral negative SLN were similar to that of node-negative patients who underwent bilateral pelvic lymphadenectomy, $85.1 \%$ vs. $80.4 \%, \mathrm{P}=0.24$ respectively (106). However, both of these studies were not designed to demonstrate the noninferiority of SLN biopsy in terms of survival.

Therefore, prospective clinical trials with large cohort are mandatory to validate the oncologic safety of SLN biopsy alone in cervical cancer. The results of the LACC trial have proven that only randomized clinical trial thoroughly conducted can evaluate medical and therapeutic techniques $(107,108)$.

To date, three ongoing prospective clinical trials aim to assess the oncological outcomes of SLN biopsy in early stage cervical cancer: the SENTIX trial (109), the PHENIX trial (110) and the SENTICOL III trial (111). The SENTIX trial (NCT02494063) is a prospective multicentric observational trial which aims to assess the 2 -year recurrence rate after SLN biopsy alone. The PHENIX trial (NCT02642471) is multi-center randomized controlled trial which aim to compare the oncological outcomes of SLN biopsy with pelvic lymphadenectomy in patients with (2-year DFS) and without (3-year DFS) SLN metastasis. The SENTICOL III trial is a prospective multicentric randomized trial which aims to compare 3-year disease-free survival between SLN biopsy alone and SLN biopsy with pelvic lymphadenectomy.

\section{Conclusions}

The SLN biopsy is a sturdy alternative to systematic full pelvic lymphadenectomy for lymph node staging in early-stage cervical cancer. This technique illustrates the type of targeted and precise surgery and is drawn in the dynamic of less aggressive management of cervical cancer. In regard with the abundant literature, there is a trend in the acceptance of SLN biopsy in current clinical practice. In addition to a decreased morbidity, SLN provides more precise anatomic mapping and accurate histologic data. The 3 ongoing prospective studies mentioned above will offer decisive data that will definitively validate SLN biopsy for lymph node staging in early-stage cervical cancer.

\section{Acknowledgments}

Funding: None.

\section{Footnote}

Provenance and Peer Review: This article was commissioned by the Guest Editors (Denis Querleu and Cherif Akladios) for the series "Sentinel Lymph Node Biopsy in Gynecologic Cancer" published in Chinese Clinical Oncology. The article was sent for external peer review.

Conflicts of Interest: All authors have completed the ICMJE uniform disclosure form (available at http://dx.doi. org/10.21037/cco-21-5). The series "Sentinel Lymph Node Biopsy in Gynecologic Cancer" was commissioned by the editorial office without any funding or sponsorship. The authors have no other conflicts of interest to declare.

Etbical Statement: The authors are accountable for all aspects of the work in ensuring that questions related to the accuracy or integrity of any part of the work are appropriately investigated and resolved.

Open Access Statement: This is an Open Access article distributed in accordance with the Creative Commons Attribution-NonCommercial-NoDerivs 4.0 International License (CC BY-NC-ND 4.0), which permits the noncommercial replication and distribution of the article with the strict proviso that no changes or edits are made and the original work is properly cited (including links to both the formal publication through the relevant DOI and the license). See: https://creativecommons.org/licenses/by-nc-nd/4.0/.

\section{References}

1. Torre LA, Bray F, Siegel RL, et al. Global cancer statistics, 2012. CA Cancer J Clin 2015;65:87-108.

2. Bhatla N, Aoki D, Sharma DN, et al. Cancer of the cervix uteri. Int J Gynaecol Obstet 2018;143 Suppl 2:22-36.

3. Paavonen J, Naud P, Salmerón J, et al. Efficacy of human papillomavirus (HPV)-16/18 AS04-adjuvanted vaccine against cervical infection and precancer caused by oncogenic HPV types (PATRICIA): final analysis of a double-blind, randomised study in young women. Lancet 2009;374:301-14.

4. Rob L, Halaska M, Robova H. Nerve-sparing and individually tailored surgery for cervical cancer. Lancet Oncol 2010;11:292-301. 
5. Guani B, Dorez M, Magaud L, et al. Impact of micrometastasis or isolated tumor cells on recurrence and survival in patients with early cervical cancer: SENTICOL Trial. Int J Gynecol Cancer 2019;29:447-52.

6. Ramirez PT, Frumovitz M, Pareja R, et al. Minimally Invasive versus Abdominal Radical Hysterectomy for Cervical Cancer. N Engl J Med 2018;379:1895-904.

7. Sartori E, Tisi G, Chiudinelli F, et al. Early stage cervical cancer: adjuvant treatment in negative lymph node cases. Gynecol Oncol 2007;107:S170-4.

8. Bhatla N, Berek JS, Cuello Fredes M, et al. Revised FIGO staging for carcinoma of the cervix uteri. Int J Gynaecol Obstet 2019;145:129-35.

9. Cibula D, Pötter R, Planchamp F, et al. The European Society of Gynaecological Oncology/European Society for Radiotherapy and Oncology/European Society of Pathology Guidelines for the Management of Patients With Cervical Cancer. Int J Gynecol Cancer 2018;28:641.

10. Koh WJ, Abu-Rustum NR, Bean S, et al. Cervical Cancer, Version 3.2019, NCCN Clinical Practice Guidelines in Oncology. J Natl Compr Canc Netw 2019;17:64-84.

11. Mathevet P, Lecuru F, Magaud L, et al. Sentinel lymph node biopsy for early cervical cancer: Results of a randomized prospective, multicenter study (Senticol 2) comparing adding pelvic lymph node dissection vs sentinel node biopsy only. Gynecol Oncol 2017;145:2-3.

12. Niikura H, Okamoto S, Otsuki T, et al. Prospective study of sentinel lymph node biopsy without further pelvic lymphadenectomy in patients with sentinel lymph node-negative cervical cancer"Int J Gynecol Cancer 2012;22:1244-50.

13. Veronesi U, Paganelli G, Viale G, et al. A randomized comparison of sentinel-node biopsy with routine axillary dissection in breast cancer. N Engl J Med 2003;349:546-53.

14. Van der Zee AGJ, Oonk MH, De Hullu JA, et al. Sentinel Node Dissection Is Safe in the Treatment of Early-Stage Vulvar Cancer. J Clin Oncol 2008;26:884-9.

15. Plante M, Renaud MC, Têtu B, et al. Laparoscopic sentinel node mapping in early-stage cervical cancer. Gynecol Oncol 2003;91:494-503.

16. Altgassen C, Hertel H, Brandstädt A, et al. Multicenter validation study of the sentinel lymph node concept in cervical cancer: AGO Study Group. J Clin Oncol 2008;26:2943-51.

17. Cormier B, Diaz JP, Shih K, et al. Establishing a sentinel lymph node mapping algorithm for the treatment of early cervical cancer. Gynecol Oncol 2011;122:275-80.

18. Tax C, Rovers MM, de Graaf C, et al. The sentinel node procedure in early stage cervical cancer, taking the next step; a diagnostic review. Gynecol Oncol 2015;139:559-67.

19. Brar H, Hogen L, Covens A. Cost-effectiveness of sentinel node biopsy and pathological ultrastaging in patients with early-stage cervical cancer. Cancer 2017;123:1751-9.

20. Cibula D, McCluggage WG. Sentinel lymph node (SLN) concept in cervical cancer: Current limitations and unanswered questions. Gynecol Oncol 2019;152:202-7.

21. Burghardt E, Pickel H. Local spread and lymph node involvement in cervical cancer. Obstet Gynecol 1978;52:138-45.

22. Inoue T, Morita K. The prognostic significance of number of positive nodes in cervical carcinoma stages IB, IIA, and IIB. Cancer 1990;65:1923-7.

23. Benedetti-Panici P, Maneschi F, Scambia G, et al. Lymphatic spread of cervical cancer: an anatomical and pathological study based on 225 radical hysterectomies with systematic pelvic and aortic lymphadenectomy. Gynecol Oncol 1996;62:19-24.

24. Euscher ED, Malpica A, Atkinson EN, et al. Ultrastaging improves detection of metastases in sentinel lymph nodes of uterine cervix squamous cell carcinoma. Am J Surg Pathol 2008;32:1336-43.

25. Horn LC, Hentschel B, Fischer U, et al. Detection of micrometastases in pelvic lymph nodes in patients with carcinoma of the cervix uteri using step sectioning: Frequency, topographic distribution and prognostic impact. Gynecol Oncol 2008;111:276-81.

26. Guani B, Balaya V, Magaud L, et al. The Clinical Impact of Low-Volume Lymph Nodal Metastases in Early-Stage Cervical Cancer: The Senticol 1 and Senticol 2 Trials. Cancers 2020;12:1061.

27. Gortzak-Uzan L, Jimenez W, Nofech-Mozes S, et al. Sentinel lymph node biopsy vs. pelvic lymphadenectomy in early stage cervical cancer: is it time to change the gold standard? Gynecol Oncol 2010;116:28-32.

28. Hancke K, Heilmann V, Straka P, et al. Pretreatment staging of cervical cancer: is imaging better than palpation?: Role of CT and MRI in preoperative staging of cervical cancer: single institution results for 255 patients. Ann Surg Oncol 2008;15:2856-61.

29. Marnitz S, Tsunoda AT, Martus P, et al. Surgical versus clinical staging prior to primary chemoradiation in patients with cervical cancer FIGO stages IIB-IVA: oncologic results of a prospective randomized international multicenter (Uterus-11) intergroup study. Int J Gynecol Cancer 2020;30:1855-61.

30. Bader AA, Winter R, Haas J, et al. Where to look for 
the sentinel lymph node in cervical cancer. Am J Obstet Gynecol 2007;197:678.e1-7.

31. Cibula D, Kocian R, Plaikner A, et al. Sentinel lymph node mapping and intraoperative assessment in a prospective, international, multicentre, observational trial of patients with cervical cancer: The SENTIX trial. Eur J Cancer 2020;137:69-80.

32. Lennox GK, Covens A. Can sentinel lymph node biopsy replace pelvic lymphadenectomy for early cervical cancer? Gynecol Oncol 2017;144:16-20.

33. Balaya V, Mathevet $\mathrm{P}$, Magaud L, et al. Predictive factors of unexpected lymphatic drainage pathways in early-stage cervical cancer. Gynecol Oncol 2019;154:102-9.

34. Kadkhodayan S, Hasanzadeh M, Treglia G, et al. Sentinel node biopsy for lymph nodal staging of uterine cervix cancer: a systematic review and meta-analysis of the pertinent literature. Eur J Surg Oncol 2015;41:1-20.

35. Buda A, Papadia A, Zapardiel I, et al. From Conventional Radiotracer Tc-99(m) with Blue Dye to Indocyanine Green Fluorescence: A Comparison of Methods Towards Optimization of Sentinel Lymph Node Mapping in Early Stage Cervical Cancer for a Laparoscopic Approach. Ann Surg Oncol 2016;23:2959-65.

36. Bats AS, Frati A, Froissart M, et al. Feasibility and performance of lymphoscintigraphy in sentinel lymph node biopsy for early cervical cancer: results of the prospective multicenter SENTICOL study. Ann Nucl Med 2015;29:63-70.

37. Bats AS, Frati A, Mathevet P, et al. Contribution of lymphoscintigraphy to intraoperative sentinel lymph node detection in early cervical cancer: Analysis of the prospective multicenter SENTICOL cohort. Gynecol Oncol 2015;137:264-9.

38. Martínez A, Zerdoud S, Mery E, et al. Hybrid imaging by SPECT/CT for sentinel lymph node detection in patients with cancer of the uterine cervix. Gynecol Oncol 2010;119:431-5.

39. Backes FJ, Cohen D, Salani R, et al. Prospective clinical trial of robotic sentinel lymph node assessment with isosulfane blue (ISB) and indocyanine green (ICG) in endometrial cancer and the impact of ultrastaging (NCT01818739). Gynecol Oncol 2019;153:496-9.

40. Frumovitz M, Euscher ED, Deavers MT, et al. "Triple injection" lymphatic mapping technique to determine if parametrial nodes are the true sentinel lymph nodes in women with cervical cancer. Gynecol Oncol 2012;127:467-71.

41. Persson J, Geppert B, Lönnerfors C, et al. Description of a reproducible anatomically based surgical algorithm for detection of pelvic sentinel lymph nodes in endometrial cancer. Gynecol Oncol 2017;147:120-5.

42. Balaya V, Bresset A, Guani B, et al. Risk factors for failure of bilateral sentinel lymph node mapping in early-stage cervical cancer. Gynecol Oncol 2020;156:93-9.

43. Body N, Grégoire J, Renaud MC, et al. Tips and tricks to improve sentinel lymph node mapping with Indocyanin green in endometrial cancer. Gynecol Oncol 2018;150:267-73.

44. Cibula D, Kuzel D, Sláma J, et al. Sentinel node (SLN) biopsy in the management of locally advanced cervical cancer. Gynecol Oncol 2009;115:46-50.

45. Dostálek L, Zikan M, Fischerova D, et al. SLN biopsy in cervical cancer patients with tumors larger than $2 \mathrm{~cm}$ and 4cm. Gynecol Oncol 2018;148:456-60.

46. Marnitz S, Köhler C, Bongardt S, et al. Topographic distribution of sentinel lymph nodes in patients with cervical cancer. Gynecol Oncol 2006;103:35-44.

47. Ouldamer L, Marret H, Acker O, et al. Unusual localizations of sentinel lymph nodes in early stage cervical cancer: a review. Surg Oncol 2012;21:e153-7.

48. Balaya V, Guani B, Magaud L, et al. Surgical algorithm for sentinel lymph nodes detection in early-stage cervical cancer. In: Plenary Session 1: Best Oral Communications Cervical Cancer. Int J Gynaecol Cancer 2019;29:A3.

49. Lécuru F, Bats AS, Bensaid C, et al. Sentinel lymph node in low stage cervical cancers. Current data. Quality assurance. Prospects. Bull Cancer (Paris) 2014;101:349-53.

50. Deng X, Zhang Y, Li D, et al. Abdominal radical trachelectomy guided by sentinel lymph node biopsy for stage IB1 cervical cancer with tumors $>2 \mathrm{~cm}$. Oncotarget 2017;8:3422-9.

51. Khoury-Collado F, Glaser GE, Zivanovic O, et al. Improving sentinel lymph node detection rates in endometrial cancer: how many cases are needed? Gynecol Oncol 2009;115:453-5.

52. Salvo G, Ramirez PT, Levenback CF, et al. Sensitivity and negative predictive value for sentinel lymph node biopsy in women with early-stage cervical cancer. Gynecol Oncol 2017;145:96-101.

53. Querleu D, Planchamp F, Chiva L, et al. European Society of Gynaecologic Oncology Quality Indicators for Advanced Ovarian Cancer Surgery. Int J Gynecol Cancer 2016;26:1354.

54. Matsuo K, Shimada M, Yamaguchi S, et al. Association of Radical Hysterectomy Surgical Volume and Survival for Early-Stage Cervical Cancer. Obstet Gynecol 
2019;133:1086-98.

55. Rossi EC, Ivanova A, Boggess JF. Robotically assisted fluorescence-guided lymph node mapping with ICG for gynecologic malignancies: a feasibility study. Gynecol Oncol 2012;124:78-82.

56. Ruscito I, Gasparri ML, Braicu EI, et al. Sentinel Node Mapping in Cervical and Endometrial Cancer: Indocyanine Green Versus Other Conventional Dyes-A Meta-Analysis. Ann Surg Oncol 2016;23:3749-56.

57. Eriksson AGZ, Montovano M, Beavis A, et al. Impact of Obesity on Sentinel Lymph Node Mapping in Patients with Newly Diagnosed Uterine Cancer Undergoing Robotic Surgery. Ann Surg Oncol 2016;23:2522-8.

58. Taşkın S, Sarı ME, Altın D, et al. Risk factors for failure of sentinel lymph node mapping using indocyanine green/ near-infrared fluorescent imaging in endometrial cancer. Arch Gynecol Obstet 2019;299:1667-72.

59. Rozenholc A, Samouelian V, Warkus T, et al. Green versus blue: Randomized controlled trial comparing indocyanine green with methylene blue for sentinel lymph node detection in endometrial cancer. Gynecol Oncol 2019;153:500-4.

60. Frumovitz M, Plante M, Lee PS, et al. The FILM Trial: A randomized phase III multicenter study assessing near infrared fluorescence in the identification of sentinel lymph nodes (SLN). Gynecol Oncol 2018;149:7.

61. Buda A, Crivellaro C, Elisei F, et al. Impact of Indocyanine Green for Sentinel Lymph Node Mapping in Early Stage Endometrial and Cervical Cancer: Comparison with Conventional Radiotracer (99m)Tc and/or Blue Dye. Ann Surg Oncol 2016;23:2183-91.

62. Bricou A, Barranger E, Uzan S, et al. Anaphylactic shock during the sentinel lymph node procedure for cervical cancer. Gynecol Oncol 2009;114:375-6.

63. Hope-Ross M, Yannuzzi LA, Gragoudas ES, et al. Adverse reactions due to indocyanine green. Ophthalmology 1994;101:529-33.

64. Bats AS, Mathevet P, Buenerd A, et al. The sentinel node technique detects unexpected drainage pathways and allows nodal ultrastaging in early cervical cancer: insights from the multicenter prospective SENTICOL study. Ann Surg Oncol 2013;20:413-22.

65. Takeshita S, Todo Y, Okamoto K, et al. Incidence of metastasis in circumflex iliac nodes distal to the external iliac nodes in cervical cancer. J Gynecol Oncol 2016;27:e42.

66. Höckel M, Horn L-C, Tetsch E, Einenkel J. Pattern analysis of regional spread and therapeutic lymph node dissection in cervical cancer based on ontogenetic anatomy. Gynecol Oncol 2012;125:168-74.

67. Lécuru F, Neji K, Robin F, et al. Drainage lymphatique de l'utérus. J Gynécologie Obstétrique Biol Reprod 2008;26:418.

68. Levenback C, van der Zee, AGJ, et al. Clinical lymphatic mapping of gynecologic cancer. London: CRC Press, 2004.

69. Ercoli A, Delmas V, Iannone V, et al. The lymphatic drainage of the uterine cervix in adult fresh cadavers: anatomy and surgical implications. Eur J Surg Oncol 2010;36:298-303.

70. Kraima AC, Derks M, Smit NN, et al. Lymphatic drainage pathways from the cervix uteri: implications for radical hysterectomy? Gynecol Oncol 2014;132:107-13.

71. Geppert B, Lönnerfors C, Bollino M, et al. A study on uterine lymphatic anatomy for standardization of pelvic sentinel lymph node detection in endometrial cancer. Gynecol Oncol 2017;145:256-61.

72. Red-Horse K. Lymphatic vessel dynamics in the uterine wall. Placenta 2008;29 Suppl A:S55-9.

73. Volchek M, Girling JE, Lash GE, et al. Lymphatics in the human endometrium disappear during decidualization. Hum Reprod 2010;25:2455-64.

74. Lécuru F, Mathevet P, Querleu D, et al. Bilateral negative sentinel nodes accurately predict absence of lymph node metastasis in early cervical cancer: results of the SENTICOL study. J Clin Oncol 2011;29:1686-91.

75. Popa I, Plante M, Renaud MC, et al. Negative sentinel lymph node accurately predicts negative status of pelvic lymph nodes in uterine cervix carcinoma. Gynecol Oncol 2006;103:649-53.

76. Leveuf J, Godard H. Les lymphatiques de l'uterus. Rev Chir 1923;61:219-48.

77. Cibula D, Abu-Rustum NR, Dusek L, et al. Bilateral ultrastaging of sentinel lymph node in cervical cancer: Lowering the false-negative rate and improving the detection of micrometastasis. Gynecol Oncol 2012;127:462-6.

78. Balaya V, Bresset A, Guani B, et al. Pre-operative surgical algorithm: sentinel lymph node biopsy as predictor of parametrial involvement in early-stage cervical cancer. Int J Gynecol Cancer 2020;30:1317-25.

79. Benoit L, Balaya V, Guani B, et al. Nomogram Predicting the Likelihood of Parametrial Involvement in EarlyStage Cervical Cancer: Avoiding Unjustified Radical Hysterectomies. J Clin Med 2020;9:2121.

80. Dostalek L, Runnebaum I, Raspagliesi F, et al. Oncologic outcome after completing or abandoning (radical) 
hysterectomy in patients with cervical cancer and intraoperative detection of lymph node positivity; ABRAX (ABandoning RAd hyst in cerviX cancer). Int J Gynecol Cancer 2020;30:261-4.

81. Dostalek L, Slama J, Fisherova D, et al. Impact of sentinel lymph node frozen section evaluation to avoid combined treatment in early-stage cervical cancer. Int J Gynecol Cancer 2020;30:744-8. Erratum in: Int J Gynecol Cancer 2020;30:1087.

82. Bentivegna E, Gouy S, Maulard A, et al. Oncological outcomes after fertility-sparing surgery for cervical cancer: a systematic review. Lancet Oncol 2016;17:e240-53.

83. Fader AN, Edwards RP, Cost M, et al. Sentinel lymph node biopsy in early-stage cervical cancer: utility of intraoperative versus postoperative assessment. Gynecol Oncol 2008;111:13-7.

84. Slama J, Dundr P, Dusek L, et al. High false negative rate of frozen section examination of sentinel lymph nodes in patients with cervical cancer. Gynecol Oncol 2013;129:384-8.

85. Martínez A, Mery E, Filleron T, et al. Accuracy of intraoperative pathological examination of SLN in cervical cancer. Gynecol Oncol 2013;130:525-9.

86. Sonoda K, Yahata H, Okugawa K, et al. Value of Intraoperative Cytological and Pathological Sentinel Lymph Node Diagnosis in Fertility-Sparing Trachelectomy for Early-Stage Cervical Cancer. Oncology 2018;94:92-8.

87. Papadia A, Morosi C, Wang J, et al. SLN mapping in early-stage cervical cancer as a minimal-invasive triaging tool for multimodal treatment. Eur J Surg Oncol 2019;45:679-83.

88. Rychlik A, Angeles MA, Migliorelli F, et al. Frozen section examination of sentinel lymph nodes can be used as a decisional tool in the surgical management of early cervical cancer. Int J Gynecol Cancer 2020;30:358-63.

89. Roy M, Bouchard-Fortier G, Popa I, et al. Value of sentinel node mapping in cancer of the cervix. Gynecol Oncol 2011;122:269-74.

90. Balaya V, Guani B, Benoit L, et al. Diagnostic value of frozen section examination of sentinel lymph nodes in early-stage cervical cancer at the time of ultrastaging. Gynecol Oncol 2020;158:576-83.

91. Okamoto S, Niikura H, Nakabayashi K, et al. Detection of sentinel lymph node metastases in cervical cancer: assessment of KRT19 mRNA in the one-step nucleic acid amplification (OSNA) method. Gynecol Oncol 2013;130:530-6.
92. Dürst M, Hoyer H, Altgassen C, et al. Prognostic value of HPV-mRNA in sentinel lymph nodes of cervical cancer patients with pN0-status. Oncotarget 2015;6:23015-25.

93. Bizzarri N, Pedone Anchora L, Zannoni GF, et al. Role of one-step nucleic acid amplification (OSNA) to detect sentinel lymph node low-volume metastasis in early-stage cervical cancer. Int J Gynecol Cancer 2020;30:364-71.

94. Mathevet P, Guani B, Ciobanu A, et al. Histopathologic Validation of the Sentinel Node Technique for Early-Stage Cervical Cancer Patients. Ann Surg Oncol 2020. [Epub ahead of print]. doi: 10.1245/s10434-020-09328-2.

95. Santoro A, Angelico G, Inzani F, et al. Standard ultrastaging compared to one-step nucleic acid amplification (OSNA) for the detection of sentinel lymph node metastases in early stage cervical cancer. Int J Gynecol Cancer 2020;30:1871-7.

96. Balsat C, Blacher S, Herfs $M$, et al. A specific immune and lymphatic profile characterizes the pre-metastatic state of the sentinel lymph node in patients with early cervical cancer. Oncoimmunology 2017;6:e1265718.

97. Corrigendum to "Revised FIGO staging for carcinoma of the cervix uteri". Int J Gynaecol Obstet 2019;147:279-80. Erratum for: Int J Gynaecol Obstet 2019;145:129-135.

98. Delomenie M, Bonsang-Kitzis H, Bats AS, et al. The clinical implication of lymph nodes micrometastases and isolated tumor cells in patients with cervical cancer: A systematic review. Eur J Obstet Gynecol Reprod Biol 2019;241:71-6.

99. Cibula D, Abu-Rustum NR, Dusek L, et al. Prognostic significance of low volume sentinel lymph node disease in early-stage cervical cancer. Gynecol Oncol 2012;124:496-501.

100. Colturato LF, Signorini Filho RC, Fernandes RCM, et al. Lymph node micrometastases in initial stage cervical cancer and tumoral recurrence. Int J Gynaecol Obstet 2016;133:69-75.

101. Nica A, Gien LT, Ferguson SE, et al. Does small volume metastatic lymph node disease affect long-term prognosis in early cervical cancer? Int J Gynecol Cancer 2020;30:285-90.

102.Abu-Rustum NR, Barakat RR. Observations on the role of circumflex iliac node resection and the etiology of lower extremity lymphedema following pelvic lymphadenectomy for gynecologic malignancy. Gynecol Oncol 2007;106:4-5.

103. Kuroda K, Yamamoto Y, Yanagisawa M, et al. Risk factors and a prediction model for lower limb lymphedema following lymphadenectomy in gynecologic cancer: a hospital-based retrospective cohort study. BMC Womens 
Health 2017;17:50.

104.Zikan M, Fischerova D, Pinkavova I, et al. A prospective study examining the incidence of asymptomatic and symptomatic lymphoceles following lymphadenectomy in patients with gynecological cancer. Gynecol Oncol 2015;137:291-8.

105. Gianoni M, Mathevet P, Uzan C, et al. Does the Sentinel Lymph Node Sampling Alone Improve Quality of Life in Early Cervical Cancer Management? Front Surg 2020;7:31.

106. Balaya V, Guani B, Magaud L, et al. ong-term oncological safety of sentinel lymph node biopsy in early-stage cervical cancer. J Clin Oncol 2020;38:6006.

107.Melamed A, Rauh-Hain JA, Ramirez PT. Minimally Invasive Radical Hysterectomy for Cervical Cancer: When Adoption of a Novel Treatment Precedes Prospective, Randomized Evidence. J Clin Oncol 2019;37:3069-74.

Cite this article as: Balaya V, Guani B, Pache B, Durand YG, Bonsang-Kitzis H, Ngô C, Mathevet P, Lécuru F. Sentinel lymph node in cervical cancer: time to move forward. Chin Clin Oncol 2021;10(2):18. doi: 10.21037/cco-21-5
108. Lecuru F, Osdoit M, Balaya V, et al. Quality assurance in surgical trials: An improvement is needed. Gynecol Oncol 2020;157:570-1.

109. Cibula D, Dusek J, Jarkovsky J, et al. A prospective multicenter trial on sentinel lymph node biopsy in patients with early-stage cervical cancer (SENTIX). Int J Gynecol Cancer 2019;29:212-5.

110. Tu H, Huang H, Xian B, et al. Sentinel lymph node biopsy versus pelvic lymphadenectomy in early-stage cervical cancer: a multi-center randomized trial (PHENIX/CSEM 010). Int J Gynecol Cancer 2020;30:1829-33.

111.Lecuru FR, McCormack M, Hillemanns P, et al. SENTICOL III: an international validation study of sentinel node biopsy in early cervical cancer. A GINECO, ENGOT, GCIG and multicenter study. Int J Gynecol Cancer 2019;29:829-34. 